\title{
ON DIFFERENTIAL GEOMETRY INTRINSICALLY CONNECTED WITH A SURFACE ELEMENT OF PROJECTIVE ARC LENGTH
}

BY

\author{
P. O. BELL
}

1. Introduction. Grove [1] has proved the existence of a remarkable class of congruences covariantly related to a surface, each congruence of which is a suitable projective substitute for the normal congruence. He has also shown that there is uniquely associated with a general one of these congruences a canonical form for the differential equations of the surface. The line of a general one of these congruences which passes through a point of the surface he has called the $R$-conjugate line at the point. The projective normal of Fubini is a special case of the $R$-conjugate line, and the associated canonical form is Fubini's canonical form.

The author [1] has presented two geometric characterizations for the $R$-conjugate line at a point of the surface, one of which exhibits this line as the cusp axis of the point with respect to the extremal curves of the integral invariant $I=\int\left(2 R v^{\prime}\right)^{1 / 2} d u$. These extremal curves were characterized geometrically, but a geometric interpretation was not obtained for the intrinsic relation which was known to exist between the integral invariant $I$ and the arc along which it is extended. The element defined by $d s=\left(2 R v^{\prime}\right)^{1 / 2} d u$ is a generalization of Fubini's element of projective arc length. In the present paper a geometric interpretation of this general element is developed and used as a basis for the formulation of such an interpretation of its integral $I$. These interpretations form a geometric foundation for a detailed investigation of the properties of the surface which are intrinsically connected with a generalized element of projective arc length. Such an investigation is initiated in this paper.

The method employed here makes extensive use of intrinsic differentiation, differentiation along a curve with respect to projective arc length of the curve. This method consists of : first, the use of intrinsic differentiation to obtain expressions for geometric concepts, and secondly, the application of a canonical system of differential equations to reinterpret these expressions.

A euclidean geometric characterization has been obtained by Abramescu [1] for the tangents of Darboux at a point of a surface. In the present paper a projective generalization of an extension of this characterization is obtained. This results from the removal of certain hypothetical restrictions which have

Presented to the Society, December 30,$1940 ; \S 6$ and $\$ 7$ contain generalizations of results presented September 7, 1939, under the title Projective invariants of a curve on a surface; received by the editors October 4, 1940. 
been placed on the base curves of the characterization and the replacement of the euclidean element of arc length by the generalized element of projective arc length.

In $\$ 5$ the associate conjugate net is defined and a characteristic geometric property is derived for it. Similar characteristic properties are obtained for conjugate tangents of a surface, the tangents of Darboux, and the tangents of Segre.

An important sequence of covariant points is geometrically determined in $\S 6$. The first three points of this sequence are used in $\$ 7$ to obtain geometric characterizations for the following differential invariants of a general curve of an arbitrary family on a surface: a generalization of Fubini's asymptotic curvature, a generalization of Bompiani's projective curvature, and a projective torsion which is introduced in this paper.

In the last section applications are made to three important special $R$-conjugate lines. A unique "metric" invariant integral and a corresponding canonical form for the differential equations of the surface are found to be associated with the normal. The normal to the surface at a point is, finally, characterized as the cusp axis of the point with respect to the extremal curves of the associated integral.

2. The fundamental canonical form. If the asymptotic net on a surface $S$ in ordinary space is chosen as parametric, the four homogeneous coordinates $y^{(i)},(i=1,2,3,4)$, of a general point $y$ of $S$ may be taken to be solutions of differential equations in Wilczynski's (so-called) canonical form

$$
y_{u u}+2 b y_{v}+f y=0, \quad y_{v v}+2 a^{\prime} y_{u}+g y=0 .
$$

Consider the parametric vector equations

$$
\begin{gathered}
\dot{y}=y(u, v), \quad \rho=y_{u}-\beta y, \quad \sigma=y_{v}-\alpha y, \\
\tau=y_{u v}-\alpha y_{u}-\beta y_{v}+\alpha \beta y
\end{gathered}
$$

where $\alpha$ and $\beta$ are arbitrary analytic functions of $u$ and $v$. Equations (2.2) define general homogeneous coordinates of four points which we denote by $y, \rho, \sigma$ and $\tau$ when no confusion can arise. The line $l$ joining the points $\rho, \sigma$ is, according to Wilczynski's classification $\left({ }^{1}\right)$, an arbitrary line of the first kind and generates a congruence of the first kind as $y$ moves over $S$. The reciprocal with respect to $S$ at $y$ of the line $l$ is the line $l^{\prime}$ which joins the points $y, \tau$. The line $l^{\prime}$ and congruence generated by $l^{\prime}$ as $y$ moves over $S$ are said to be of the second kind. If the functions $\alpha, \beta$ are suitably chosen, the points $y, \rho, \sigma$ and $\tau$ become covariant points and the congruences generated by $l$ and $l^{\prime}$ become covariant congruences.

The most general transformation of independent variables $u, v$ which

(1) See Wilczynski [2, p. 95]. Green [1, p. 114] used this means of classifying his canonical edges of the first and second kinds. 
transforms $u$-curves into $\bar{u}$-curves and $v$-curves into $\bar{v}$-curves is

$$
\bar{u}=\bar{u}(u), \quad \bar{v}=\bar{v}(v) .
$$

If (2.3) is performed on system (2.1), a new system is obtained whose coefficients are

$$
\begin{aligned}
& \bar{a}=\bar{u}_{u u} / 2 \bar{u}_{u}^{2}, \quad \bar{b}=b \bar{v}_{v} / \bar{u}_{u}^{2}, \quad \bar{f}=f / \bar{u}_{u}^{2}, \\
& \bar{a}^{\prime}=a^{\prime} \bar{u}_{u} / \bar{v}_{v}^{2}, \quad \bar{b}^{\prime}=\bar{v}_{v v} / 2 \bar{v}_{v}^{2}, \quad \bar{g}=g / \bar{v}_{v}^{2},
\end{aligned}
$$

wherein $2 \bar{a}$ is the coefficient of $y_{\bar{u}}$ in the first equation and $2 \bar{b}^{\prime}$ is the coefficient of $y_{i}$ in the second equation of the system.

The transformation

$$
y=\mu \bar{y},
$$

where $\mu$ is an analytic function of $u$, $v$, leaves the surface undisturbed and maintains the parametric character of the asymptotic net.

Consider with Grove $[1$, p. 583] an invariant $R$ of the surface-in our notation a function of the coefficients of (2.1) which is an invariant of (2.5)with the following properties: (a) $R \neq 0$, (b) the transform $\bar{R}$ of $R$ by (2.3) is $\bar{R}=R / \bar{u}_{u} \bar{v}_{v}$. The transform of $d u d v$ by (2.3) is given by $d \bar{u} d \bar{v}=\bar{u}_{u} \bar{v}_{v} d u d v$. Hence the quadratic form

$$
(d s)^{2}=2 R d u d v
$$

is absolutely invariant under (2.3) and (2.5).

The most general transformation which preserves the parametric character of the asymptotic net and at the same time maintains Wilczynski's canonical form $(2.1)$ is $\left({ }^{2}\right)$

$$
y=c \bar{y} /\left(\bar{u}_{u} \bar{v}_{v}\right)^{1 / 2}, \quad \bar{u}=\bar{u}(u), \quad \bar{v}=\bar{v}(v), \quad c=\text { const. }
$$

Consider the points $x_{u}, x_{v}$ where $x$ is defined by $x=R^{h} y, h=$ const. From property (b) and equations (2.7) we obtain

$$
\left(R^{h} y\right)_{u}=\bar{u}_{u}\left(\left[\bar{u}_{u} \bar{v}_{v} \bar{R}\right]^{h} \bar{y} /\left[\bar{u}_{u} \bar{v}_{v}\right]^{1 / 2}\right)_{\bar{u}}, \quad\left(R^{h} y\right)_{v}=\bar{v}_{v}\left(\left[\bar{u}_{u} \bar{v}_{v} \bar{R}\right]^{h} \bar{y} /\left[\bar{u}_{u} \bar{v}_{v}\right]^{1 / 2}\right)_{\bar{v}} .
$$

These relations show clearly that $x_{u}, x_{v}$ are covariant points if and only if $h=1 / 2$. The transformation $y=x / R^{1 / 2}$ transforms system (2.1) into the general canonical form of Grove:

$$
\begin{aligned}
& x_{u u}=p x+\theta_{u} x_{u}+\beta x_{v}, \\
& x_{v v}=q x+\gamma x_{u}+\theta_{v} x_{v},
\end{aligned}
$$

wherein $\theta=\log R, \quad \beta=-2 b, \quad \gamma=-2 a^{\prime}, \quad p=-f+b \theta_{v}+\theta_{u u} / 2-\theta_{u}^{2} / 4 \quad$ and

$\left(^{2}\right)$ See Wilczynski [1, p. 256]. 
$q=-g+a^{\prime} \theta_{u}+\theta_{v v} / 2-\theta_{v}^{2} / 4$. The points $x_{u}, x_{v}, x_{u v}$ are covariant points. The points $x_{u}, x_{v}$ are the intersections of the $R$-harmonic line with the asymptotic $u$ - and $v$-tangents to $S$ at $x$, and the point $x_{u v}$ lies on the $R$-conjugate line and is characterized geometrically in an obvious manner. The $R$-conjugate and $R$-harmonic lines are not only reciprocals with respect to $S$ at $x$ but as $x$ varies over $S$ they generate congruences which are respectively conjugate and harmonic to $S$.

The forms (2.6) and (2.9) are generalizations of Fubini's normal form and canonical forms respectively, and the $R$-conjugate line is a generalization of Fubini's projective normal. Each of these forms of Fubini corresponds, as does also the projective normal, to the selection $R=\beta \gamma$. Because of its unique association with the $R$-conjugate line, it seems appropriate to call the form (2.9) the $R$-canonical form.

3. The geometric characterization of the integral invariant. Consider any two covariant (geometrically determined) points $\omega_{1}$ and $\omega_{2}$ which are collinear with $x$ but do not lie in the tangent plane to $S$ at $x$. The general coordinates for $\omega_{1}$ and $\omega_{2}$ are given by $\omega_{1}=x_{u v}-c x_{u}-d x_{v}+r x, \omega_{2}=\omega_{1}-R x / 2$, where $c, d, r$ and $R$ are functions of $u, v$ whose values depend on the selection of $\dot{\omega}_{1}, \omega_{2}$. Referred to a local tetrahedron whose vertices are the points

$$
x, \quad \rho=x_{u}-d x, \quad \sigma=x_{v}-c x, \quad \omega_{1},
$$

the equation for a general quadric of Darboux may be easily found to be

$$
x_{2} x_{3}-x_{1} x_{4}+k x_{4}^{2}=0,
$$

where $k$ is an arbitrary function of $u, v$. The unique quadric which passes through the point $\omega_{2}$ has the equation

$$
2\left(x_{2} x_{3}-x_{1} x_{4}\right)-R x_{4}^{2}=0 .
$$

Putting $x_{1}=0$, we obtain the equation

$$
2 x_{2} x_{3}-R x_{4}^{2}=0
$$

for the cone whose vertex is the point $x$ and which passes through the conic of intersection of the plane $x_{1}=0$ with the quadric (3.2). An arbitrary generator $l_{h}^{\prime}$ of this cone joins the points $x, 2\left(\omega_{1} h+R \rho\right)+h^{2} \sigma, h=$ arbitrary const. The reciprocal of $l_{h}^{\prime}$, which we denote by $l_{h}$, joins the points $\rho+h x / 2$, $\sigma+R x / h$. The equations for $l_{h}$ are therefore

$$
2 h x_{1}-h^{2} x_{2}-2 R x_{3}=0, \quad x_{4}=0 .
$$

As $h$ is varied while $u, v$ are held constant the line $l_{h}^{\prime}$ generates the cone (3.3), and the line $l_{h}$ envelops a conic $C$ whose equation, when referred to the tri- 
angle of reference whose vertices are the points $x, \rho=x_{u}-d x, \sigma=x_{v}-c x$ may easily be found to be

$$
x_{1}^{2}-2 R x_{2} x_{3}=0 .
$$

This conic touches the asymptotic $u$-and $v$-tangents to $S$ at $x$ in the points $\rho$ and $\sigma$, in which the reciprocal of the line joining $\omega_{1}, \omega_{2}$ intersects these tangents. This important conic plays a basic role in the geometric determinations of the present paper.

An arbitrary one-parameter family of curves on $S$ is defined by the curvilinear equation

$$
d v-\lambda(u, v) d u=0
$$

where $\lambda$ is an arbitrary function of $u, v$. We shall denote by $F_{\lambda}$ the family defined by (3.6) and by $C_{\lambda}$ the curve of the family which passes through the point $x$.

The differential form

$$
d s=(2 R \lambda)^{1 / 2} d u,
$$

which corresponds to an arbitrary selection of covariant points $\omega_{1}, \omega_{2}$, is an absolute invariant under the most general transformation of independent and dependent variables maintaining the asymptotic net as parametric.

To provide a geometric interpretation for $d s$ let $Y$ denote a point on the tangent to $C_{\lambda}$ at $x$ and let $N$ and $\bar{N}$ denote the points in which this tangent intersects the conic $\mathcal{C}$. We define the non-euclidean distance from $x$ to $Y$ to be

$$
D_{x Y}=(x, \bar{N}, Y, N) / 2 .
$$

The general coordinates of $N$ and $\bar{N}$ are given by $N=\rho+\lambda \sigma+(2 R \lambda)^{1 / 2} x$, $\bar{N}=\rho+\lambda \sigma-(2 R \lambda)^{1 / 2} x$. Let $X$ denote a point near to $x$ on the curve $C_{\lambda}$, where the curvilinear coordinates of $x$ and $X$ are $(u, v)$ and $(u+\delta u, v+\delta v)$, respectively. Since $X=x(u+\delta u, v+\delta v)$ and the limit of $\delta v / \delta u$ as $\delta u$ tends to zero is $\lambda(u, v)$, for sufficiently small values of $\delta u$ the general coordinates of $X$ may be given by the expansion

$$
X=x+\left(x_{u}+\lambda x_{v}\right) \delta u+\text { terms of order } \delta u^{2} \text { at least, }
$$

wherein $x=x(u, v)$. Hence coordinates of $X$ differ only by terms of order $\delta u^{2}$ from those of the point $X_{1}$ on the tangent to $C_{\lambda}$ at $x$ given by $X_{1}=x$ $+\left(x_{u}+\lambda x_{v}\right) \delta u$. Therefore the principal parts of the infinitesimal cross ratios

$$
(x, \bar{N}, X, N), \quad\left(x, \bar{N}, X_{1}, N\right)
$$

are identical. It may be easily shown that this principal part is the absolute differential invariant (3.7).

To interpret geometrically the integral of the form (3.7) extended over a 
finite arc of $C_{\lambda}$ let $A$ and $B$ denote the end points of the arc, and let $\left(u_{0}, v_{0}\right)$ and $(u, v)$ denote the curvilinear coordinates of $A$ and $B$, respectively. Let $\epsilon$ be a positive number, and divide the $\operatorname{arc} A B$ by means of intermediate points $X_{i},(i=1,2, \cdots, n-1)$, into $n$ smaller arcs. Let the curvilinear coordinates of $X_{k}$ be $\left(u_{k}, v_{k}\right)$, where $u_{n}=u$ and $v_{n}=v$, and where

$$
\left[\left(u_{k}-u_{k-1}\right)^{2}+\left(v_{k}-v_{k-1}\right)^{2}\right]^{1 / 2} \leqq \epsilon, \quad k=1,2, \cdots, n,
$$

with $\epsilon$ tending to zero as $n$ increases without limit. Then if we put $u_{k}-u_{k-1}$ $=\delta u_{k}$ and $v_{k}-v_{k-1}=\delta v_{k}$, we have

$$
I=\int_{A B} d s=\lim _{n \rightarrow \infty} \sum_{k=1}^{n} D_{X_{k-1} X_{k}}
$$

We have therefore

TheOREM 1. The integral of the form (3.7) extended over an arc $A B$ is the limit of the sum of infinitesimal non-euclidean distances, each of which is defined at a separate point $X_{k-1}$ of the arc as one-half of the principal part of the corresponding cross ratio $\left(X_{k-1}, \bar{N}, X_{k}, N\right)$ which is geometrically determined at $X_{k-1}$.

We shall call the integral $I$ extended over an arc of $S$ projective arc length with respect to the form (3.7).

It may be observed that Fubini's element of projective arc length

$$
d s=(2 \beta \gamma \lambda)^{1 / 2} d u
$$

is a special case of (3.7).

4. The tangents of Darboux. Let $C$ be a curve of normal section of a surface $S$ (in euclidean space) through a point $x$ on $S$. Let $M_{1}, M_{2}$ be points at distances $s$ and $-s$ from $x$ measured along $C$. The line $M_{1} M_{2}$ intersects the tangent to $C$ at $x$ in a point $T_{a}$. Abramescu [1] has recently obtained the following results: The limit of $T_{a}$ as $s$ tends to zero is a point $T$ distinct from $x$. The locus of $T$ as $C$ varies through the set of normal sections is a rational cubic curve. The lines joining the point $x$ to the points of inflection of this cubic curve are the tangents of Darboux.

These results may be extended as follows. Let the curve of normal section of $S$ be replaced by an arbitrary curve $C_{\lambda}$ of $S$. Let $\bar{X}_{1}, \bar{X}_{2}$ denote the points at distances $s$ and $-s$ measured from $x$ along $C_{\lambda}$. Let $T_{s}$ denote the point in which the tangent plane to $S$ at $x$ intersects the line joining $\bar{X}_{1}, \bar{X}_{2}$. It may be easily shown that if the curves $C$ and $C_{\lambda}$ have at $x$ a common osculating plane, the limit points of $T_{a}$ and $T_{s}$ as $s$ tends to zero coincide in the point $T$. Thus the normal sections of $S$ may be replaced in the characterization by less restricted curves.

These results are capable of projective generalization as follows. Let projective arc lengths $s,-s$ be measured with respect to the form (3.7) from the point $x$ along the curve $C_{\lambda}$. Let the end points of this arc be denoted by $x(s)$, 
$x(-s)$. The line joining $x(s), x(-s)$ intersects the tangent plane to $S$ at $x$ in a point which will be denoted by $P_{s}$.

Theorem 4.1. The limit of the point $P_{s}$ as $s$ tends to zero is a point $P_{0}$, distinct from $x$, which lies on the tangent to $C_{\lambda}$ at $x$. The locus of $P_{0}$ as the direction of $C_{\lambda}$ is varied at $x$ is a rational cubic curve. The lines joining the point $x$ to the points of inflection of this cubic are the tangents of Darboux. The line joining the three points of inflection is the $R$-harmonic line (the reciprocal of the $R$-conjugate line).

To prove this theorem we shall need the expressions for the first, second and third derivatives of $x$ with respect to $s$, where $d s$ is defined by (3.7). By making use of the fundamental differential equations in the $R$-canonical form (2.4) of Grove we obtain

$$
\begin{aligned}
d x / d s & =\left(x_{u}+\lambda x_{v}\right) /(2 R \lambda)^{1 / 2}, \\
d^{2} x / d s^{2} & =\left(x_{u v}-c x_{u}-d x_{v}+n x\right) / R
\end{aligned}
$$

wherein $c, d$ and $n$ are defined by

$$
\begin{aligned}
c & =\left(\lambda^{\prime}+\theta_{v} \lambda^{2}-\theta_{u} \lambda-2 \gamma \lambda^{3}\right) / 4 \lambda^{2}, & d & =-\left(\lambda^{\prime}+2 \beta-\theta_{u} \lambda+\theta_{v} \lambda^{2}\right) / 4 \lambda, \\
n & =\left(p+q \lambda^{2}\right) / 2 \lambda, & \lambda^{\prime} & =\lambda_{u}+\lambda \lambda_{v},
\end{aligned}
$$

wherein

$$
\begin{aligned}
& A=\left(\gamma \lambda^{3}+\beta\right) / 2 \lambda, \\
& B=\theta_{u v}+\beta \gamma+\lambda \chi-c_{u}-c_{v} \lambda-b \gamma \lambda+n+c \theta_{v} \lambda, \\
& C=\lambda \theta_{u v}+\lambda \beta \gamma+\Pi-d_{u}-d_{v} \lambda-c \beta+n \lambda+d \theta_{u}, \\
& D=p_{v}+\beta q+n_{u}-c p-n \theta_{u}+\lambda\left(q_{u}+\gamma p+n_{v}-d q-n \theta_{v}\right), \\
& \chi=q+\gamma \theta_{u}+\gamma_{u}, \quad \Pi=p+\beta \theta_{v}+\beta_{v}, \quad \theta=\log R .
\end{aligned}
$$

If we take $s$ as the independent variable, for sufficiently small values of $s$ the general coordinates of $x(s)$ and $x(-s)$ are given by the expansions

$$
\begin{aligned}
x(s) & =x+(d x / d s) s+\left(d^{2} x / d s^{2}\right) s^{2} / 2+\left(d^{3} x / d s^{3}\right) s^{3} / 6+\cdots, \\
x(-s) & =x-(d x / d s) s+\left(d^{2} x / d s^{2}\right) s^{2} / 2-\left(d^{3} x / d s^{3}\right) s^{3} / 6+\cdots .
\end{aligned}
$$

The general coordinates of the point $P_{s}$ may be found by forming a linear combination of $x(s)$ and $x(-s)$ which is free from terms involving $x_{u v}$. By making use of the equations (4.1), (4.2), (4.3) and (4.4) we find that the coefficient of $x_{u v}$ in $x(s)$ is given by $s^{2} / 2 R+A \lambda s^{3} / 3(2 R \lambda)^{3 / 2}+$ terms of order $s^{4}$. Since the expansion for $x(-s)$ differs from that of $x(s)$ only in the signs of its terms of odd degree in $s$, the coefficient of $x_{u v}$ in $x(-s)$ must be given by 
$s^{2} / 2 R-A \lambda s^{3} / 3(2 R \lambda)^{3 / 2}+$ terms of order $s^{4}$. Hence the point $P_{s}$, except for terms of order $s^{5}$, is represented by the linear combination

$$
\left[1 / 2 R+A \lambda s / 3(2 R \lambda)^{3 / 2}\right] x(-s)-\left[1 / 2 R-A \lambda s / 3(2 R \lambda)^{3 / 2}\right] x(s) .
$$

Performing the operations indicated by this combination and omitting an unessential factor $s$, we find the general homogeneous coordinates of $P_{s}$ to be defined by

$$
\begin{aligned}
2 A \lambda x / 3(2 R \lambda)^{3 / 2}-x^{\prime} / R+\left[A \lambda x^{\prime \prime} / 3(2 R \lambda)^{3 / 2}\right. & \left.-x^{\prime \prime \prime} / 6 R\right] s^{2} \\
& + \text { terms of order } s^{3} \text { at least. }
\end{aligned}
$$

The limit of the point $P_{s}$ as $s$ tends to zero is the point $P_{0}$, distinct from $x$, which lies on the tangent to $C_{\lambda}$ at $x$ and whose general homogeneous coordinates (except for a non-essential factor) are given by

$$
\left(\gamma \lambda^{3}+\beta\right) x-6 \lambda x_{u}-6 \lambda^{2} x_{v} .
$$

As the direction $\lambda$ is varied while $u, v$ are held constant, the point $P_{0}$ describes the rational cubic curve whose equation is

$$
\gamma x_{3}^{3}+\beta x_{2}^{3}+6 x_{1} x_{2} x_{3}=0,
$$

when referred to the triangle whose vertices are the points $x, x_{u}$ and $x_{v}$. The three points of inflection lie on the line whose equation is $x_{1}=0$. Since the points $x_{u}, x_{v}$ also lie on this line, it is the $R$-harmonic line. The equation

$$
\gamma x_{3}^{3}+\beta x_{2}^{3}=0
$$

represents the three lines which join the point $x$ to the different points of inflection. The form of equation (4.10) identifies these lines as the tangents of Darboux. This completes the proof of the results stated in the opening paragraph of this section. fore,

If $\lambda$ satisfies the equation $\gamma \lambda^{3}+\beta=0$ it is a direction of Darboux. There-

THEOREM 4.2. The point $P_{0}$ which corresponds to a direction $\lambda$ lies on the $R$-harmonic line if and only if $\lambda$ is a direction of Darboux.

Since the coefficient of $x_{u v}$ in the form (4.4) for the coordinates of the point $d^{3} x / d s^{3}$ is $\left(\gamma \lambda^{3}+\beta\right) /(2 R \lambda)^{3 / 2}$, we have

Theorem 4.3. The point $d^{3} x / d s^{3}$, where $d s=(2 R \lambda)^{1 / 2} d u, \lambda \neq \infty$, and $R$ is an arbitrary function of $(u, v)$, lies in the tangent plane to $S$ at $x$ if and only if the direction $\lambda$ is a direction of Darboux.

5. Theorems on nets. The curvilinear differential equation of an arbitrary net $N_{\lambda, \lambda_{1}}$ on the surface $S$ can be written in the form 


$$
(d v-\lambda d u)\left(d v-\lambda_{1} d u\right)=0,
$$

where $\lambda, \lambda_{1}$ are arbitrary functions of $u$, $v$. There exists on $S$ a conjugate net uniquely determined by the net $N_{\lambda, \lambda_{1}}$, such that at each point of $S$ its tangents separate the tangents of the net $N_{\lambda, \lambda_{1}}$ harmonically. This net will be called the associate conjugate net of the net $N_{\lambda_{,} \lambda_{1}}$ (Green [2, p. 213]). Its differential equation is found to be

$$
d v^{2}-\lambda \lambda_{1} d u^{2}=0 .
$$

A remarkable characteristic property of this net may be obtained as follows. Let projective arc lengths $s,-s$ be measured with respect to the form $d s=\left(2 R v^{\prime}\right)^{1 / 2} d u$ from the point $x$ along the curves $C_{\lambda}, C_{\lambda_{1}}$. Let the end points of these arcs be denoted by $x(s), x(-s)$ and $x_{1}(s), x_{1}(-s)$, respectively. The line joining the points $x(s), x_{1}(s)$ and the line joining the points $x(s), x_{1}(-s)$ intersect the tangent plane to $S$ at $x$ in points which we denote by $Q_{s}$ and $\bar{Q}_{s}$, respectively.

TheOREM 5.1. The points $Q_{s}$ and $\bar{Q}_{s}$ approach limit points $Q_{0}$ and $\bar{Q}_{0}$, respectively, as $s$ tends to zero. The tangents to $S$ at $x$ joining $x$ to the separate points $Q_{0}$ and $\bar{Q}_{0}$ are the tangents at $x$ of the associate conjugate net of the net $N_{\lambda, \lambda_{1}}$.

To prove this theorem consider the general coordinates of the points $x(s), x_{1}(s), x_{1}(-s)$. The coordinates of $x(s)$ for small values of $s$ are given by the expansion (4.5), wherein the derivatives are defined by (4.1), (4.2) and (4.4). The coordinates of $x_{1}(s)$ are obtained from those of $x(s)$ by replacing $\lambda$ by $\lambda_{1}$. The coordinates of $x_{1}(-s)$ may be obtained from those of $x_{1}(s)$ by changing the signs of the terms containing odd exponents of $s$. The coefficient of $x_{u v}$ in $x(s)$ is given by $s^{2} / 2 R+\left(\gamma \lambda^{3}+\beta\right) s^{3} / 6(2 R \lambda)^{3 / 2}+$ terms of order $s^{4}$ at least. The coefficients of $x_{u v}$ in $x_{1}(s)$ and $x_{1}(-s)$ are (except for terms of order $\left.s^{4}\right)$ given by $s^{2} / 2 R+\left(\gamma \lambda_{1}^{3}+\beta\right) s^{3} / 6\left(2 R \lambda_{1}\right)^{3 / 2}$ and $s^{2} / 2 R-\left(\gamma \lambda_{1}^{3}+\beta\right) s^{3} / 6\left(2 R \lambda_{1}\right)^{3 / 2}$, respectively. Therefore, except for terms of order $s^{3}$ at least, the points $Q_{s}, \bar{Q}_{s}$ are given by

$$
\begin{gathered}
\left(1 / 2 R+\left[\gamma \lambda^{3}+\beta\right] s / 6[2 R \lambda]^{3 / 2}\right) x_{1}(s)-\left(1 / 2 R+\left[\gamma \lambda_{1}^{3}+\beta\right] s / 6\left[2 R \lambda_{1}\right]^{3 / 2}\right) x(s), \\
\left(1 / 2 R+\left[\gamma \lambda^{3}+\beta\right] s / 6[2 R \lambda]^{3 / 2}\right) x_{1}(-s)-\left(1 / 2 R-\left[\gamma \lambda_{1}^{3}+\beta\right] s / 6\left[2 R \lambda_{1}\right]^{3 / 2}\right) x(s),
\end{gathered}
$$

respectively. The developments for $x(s), x_{1}(s), x_{1}(-s)$ which are needed are (except for terms of order $s^{2}$ at least)

$$
\begin{aligned}
x(s) & =x+\left(x_{u}+\lambda x_{v}\right) s /(2 R \lambda)^{1 / 2}+\cdots, \\
x_{1}(s) & =x+\left(x_{u}+\lambda_{1} x_{v}\right) s /\left(2 R \lambda_{1}\right)^{1 / 2}+\cdots, \\
x_{1}(-s) & =x-\left(x_{u}+\lambda_{1} x_{v}\right) s /\left(2 R \lambda_{1}\right)^{1 / 2}+\cdots .
\end{aligned}
$$

Performing the operations indicated in the expressions for $Q_{s}, \bar{Q}_{s}$ and neglecting unessential factors, we obtain the following forms: 


$$
\begin{aligned}
& \left(\gamma \lambda_{2}-\beta / \lambda_{2}^{2}\right)\left(\lambda+\lambda_{1}+\lambda_{2}\right) x+x_{u}-\lambda_{2} x_{v}, \\
& \left(\gamma \lambda_{2}+\beta / \lambda_{2}^{2}\right)\left(\lambda+\lambda_{1}-\lambda_{2}\right) x-x_{u}-\lambda_{2} x_{v}, \quad \lambda_{2}=\left(\lambda \lambda_{1}\right)^{1 / 2},
\end{aligned}
$$

for the general coordinates of $Q_{0}$ and $\bar{Q}_{0}$, respectively. Since the directions of the lines joining $x$ to the points $Q_{0}$ and $\bar{Q}_{0}$ are, respectively, $-\left(\lambda \lambda_{1}\right)^{1 / 2}$ and $\left(\lambda \lambda_{1}\right)^{1 / 2}$, the proof of the theorem is complete.

Consider, as a special case of the net $N_{\lambda, \lambda_{1}}$, the conjugate net $N_{\lambda}$. If we replace $\lambda_{1}$ by $-\lambda$ in (5.3) and (5.4), we obtain the forms

$$
\begin{aligned}
& \left(i \gamma \lambda+\beta / \lambda^{2}\right) i \lambda x+x_{u}-i \lambda x_{v}, \\
& \left(i \gamma \lambda-\beta / \lambda^{2}\right) i \lambda x+x_{u}+i \lambda x_{v}, \quad i=(-1)^{1 / 2},
\end{aligned}
$$

for the coordinates of the associated points $Q_{0}$ and $\bar{Q}_{0}$, respectively. If, however, we replace $\lambda$ by $-\lambda_{1}$, the forms (5.5) and (5.6) are interchanged. The reason for this rather surprising fact becomes clear if we notice the effect of these replacements in the equation

$$
d s=(2 R \lambda)^{1 / 2} d u=\left(2 R \lambda_{1}\right)^{1 / 2} d u_{1}
$$

which determines at $x$ the sense of direction of $x$ along $C_{\lambda_{1}}$ which corresponds to a given sense of direction of $x$ along $C_{\lambda}$. If we put $-\lambda$ for $\lambda_{1}$ in (5.7), we have at $x$

$$
d u=i d u_{1} .
$$

If, however, we put $-\lambda_{1}$ for $\lambda$ in (5.7), we have at $x$

$$
d u=-i d u_{1} .
$$

The senses of direction defined for $x$ along $C_{\lambda_{1}}$ by (5.8) and (5.9) are, clearly, opposed to one another. Hence, the order of the determination of the points $Q_{0}, \bar{Q}_{0}$ which corresponds to (5.9) is the reverse of that which corresponds to (5.8).

The line joining the points $Q_{0}, \bar{Q}_{0}$ has the equation

$$
x_{1}+\gamma \lambda^{2} x_{2}+\beta x_{3} / \lambda^{2}=0 .
$$

It will be called the $N_{\lambda}$-associate of the $R$-harmonic line. This line intersects the $R$-harmonic line in a point of the tangent to $S$ at $x$ whose equation is

$$
\gamma \lambda^{2} x_{2}+\beta x_{3} / \lambda^{2}=0 .
$$

This tangent will be called the $N_{\lambda}$-correspondent of the tangent to $C_{\lambda}$ at $x$.

The following theorems are immediate consequences of the form of equation (5.11):

TheOREM 5.2. The $N_{\lambda}$-correspondent of the tangent to a non-asymptotic curve $C_{\lambda}$ at $x$ coincides with this tangent if and only if it is a tangent of Darboux. 
TheOREM 5.3. The tangent to a non-asymptotic curve $C_{\lambda}$ at $x$ and its $N_{\lambda}$-correspondent are conjugate tangents if and only if $\lambda$ is a direction of Segre.

If $\lambda_{1}$ is made to approach $\lambda$, the point $Q_{0}$ tends to a limit point $Q$ whose general homogeneous coordinates are found, by replacing $\lambda_{1}$ by $\lambda$ in (5.3), to be given by

$$
3\left(\gamma \lambda^{3}-\beta\right) x+\lambda x_{u}-\lambda^{2} x_{v} .
$$

Since the ratio of the coefficient of $x_{v}$ to that of $x_{u}$ in (5.12) is equal to $-\lambda$, we have

Theorem 5.4. The line joining the points $x$ and $Q$ and the tangent to $C_{\lambda}$ at $x$ are conjugate tangents.

The equation obtained by equating to zero the coefficient of $x$ in (5.12) is satisfied by the three directions of Segre. Hence we have

THEOREM 5.5. The tangent to $C_{\lambda}$ at $x$ is a tangent of Segre if and only if the associated point $Q$ is the intersection of the $R$-harmonic line with the conjugate of the tangent to $C_{\lambda}$ at $x$.

As $\lambda$ is varied while $u, v$ are held constant, the point $Q$ describes a rational cubic curve whose equation, referred to the triangle whose vertices are $x, x_{u}$, $x_{v}$, is easily found to be

$$
\gamma x_{3}^{3}+\beta x_{2}^{3}-\frac{1}{3}\left(x_{1} x_{2} x_{3}\right)=0 .
$$

This cubic has geometric properties similar to those of the cubic (4.9).

6. A sequence of covariant points. The points of the sequence $x^{(j)}=d^{i} x / d s^{i}$, $(j=1,2, \cdots)$, where $d s=(2 R \lambda)^{1 / 2} d u$, are covariant. A general one of them is intrinsically and projectively related to the curve $C_{\lambda}$ at $x$. A geometric interpretation of this relation will be presented in this section. The points $x^{(j)}-x^{(j-1)}$ and $x^{(j)}+x^{(j-1)}$ will play basic roles in this interpretation.

Let $C(j,-), C(j,+)$, and $C(j)$ denote, respectively, the curves described by the points $x^{(j)}-x^{(j-1)}, x^{(j)}+x^{(j-1)}$, and $x^{(j)}$ as $x$ moves along $C_{\lambda}$.

The point $x^{\prime}=\left(x_{u}+\lambda x_{v}\right) /(2 R \lambda)^{1 / 2}$ is, clearly, the intersection of the tangent to $C_{\lambda}$ at $x$ with the $R$-harmonic line. If the fundamental points $\omega_{1}, \omega_{2}$, $\omega_{1}-\omega_{2}=R x / 2$, are selected on the $R$-conjugate line, the associated conic $\mathcal{C}$, referred to the triangle whose vertices are $x, x_{u}, x_{v}$, has the equation (3.5). This conic intersects the tangent to $C_{\lambda}$ at $x$ in the points $x^{\prime}+x$ and $x^{\prime}-x$. The tangents at these points to the curves $C(1,-), C(1,+)$ intersect the tangent at $x^{\prime}$ to the curve $C(1)$ in the points $x^{\prime \prime}+x^{\prime}$ and $x^{\prime \prime}-x^{\prime}$. The point $x^{\prime \prime}$ is clearly the harmonic conjugate of the point $x^{\prime}$ with respect to the points $x^{\prime \prime}+x^{\prime}$ and $x^{\prime \prime}-x^{\prime}$. Similarly the tangents at $x^{\prime \prime} \pm x^{\prime}$ to the curves $C(2, \pm)$ intersect the tangent at $x^{\prime \prime}$ to the curve $C(2)$ in the points $x^{\prime \prime \prime}+x^{\prime \prime}$ and $x^{\prime \prime}-x^{\prime \prime}$. The point $x^{\prime \prime \prime}$ is the harmonic conjugate of $x^{\prime \prime}$ with respect to the points $x^{\prime \prime \prime} \pm x^{\prime \prime}$. It 
is quite clear that the entire sequence $x^{(j)} \pm x^{(j-1)},(j=1,2, \cdots)$, is determined in this way, such that $x^{(k)}$ is the harmonic conjugate of $x^{(k-1)}$ with respect to the points $x^{(k)}+x^{(k-1)}$ and $x^{(k)}-x^{(k-1)}$ for an arbitrary integer $j=k$.

The geometric locations of the points $x^{\prime \prime}$ and $x^{\prime \prime \prime}$ will help to form geometric interpretations for the second and third order differential invariants of $C_{\lambda}$ known as projective curvature and projective torsion.

7. Differential invariants of a curve on a surface. Fubini's asymptotic curvature and Bompiani's projective curvature are second order differential invariants of a curve $C_{\lambda}$ on a surface $S$. Bompiani's projective torsion of $C_{\lambda}$ is a third order differential invariant. These invariants have been defined analytically by the respective forms

$$
\begin{aligned}
\kappa_{a}= & R\left(d u \delta^{2} v-d v \delta^{2} u\right) / \phi_{2}^{3 / 2} \\
\kappa= & \kappa_{a}+\bar{\phi}_{3} / 2 \phi_{2}^{3 / 2} \\
T= & {\left[R\left(d u \delta^{3} v-d v \delta^{3} u\right) \phi_{2}-\kappa_{a} \phi_{2}^{3 / 2} \phi_{3} / 2\right.} \\
& \left.\quad+3 R\left(\beta d u^{2} \delta^{2} u-\gamma d v^{2} \delta^{2} v\right) \phi_{2}-\phi_{3} \bar{\phi}_{3} / 4+\bar{\psi}_{2} \phi_{2}^{2}-\bar{\phi}_{4} \phi_{2}\right] / \phi_{2}^{3}
\end{aligned}
$$

wherein $\phi_{2}=2 R d u d v, \phi_{3}=2 R\left(\beta d u^{3}+\gamma d v^{3}\right), \bar{\phi}_{3}=2 R\left(\beta d u^{3}-\gamma d v^{3}\right), \psi_{2}=(p+3 \beta \Psi / 2)$ $d u^{2}-(q+3 \gamma \Phi / 2) d v^{2}, \bar{\phi}_{4}=\beta R \Phi d u^{4}-\gamma R \Psi d v^{4}, \Psi=(\log \beta R)_{v}, \Phi=(\log \gamma R)_{u}, d v / d u$ $=\lambda, R=\beta \gamma$, and $\delta^{2} u, \delta^{2} v, \delta^{3} u, \delta^{3} v$ represent contravariant differentials constructed with respect to the form $\left(^{3}\right) \phi_{2}$.

Bompiani $\left(^{4}\right)$ has obtained geometric interpretations for the infinitesimal invariants $\kappa_{i} \phi_{2}^{1 / 2}, \kappa \phi_{2}^{1 / 2}$, and $T \phi_{2}^{1 / 2}$, where $\phi_{2}=2 \beta \gamma d u d v$. However he has given no geometric interpretation for $\kappa_{a}, \kappa$, or $T$.

The invariants $\kappa_{a}, \kappa, T$ are special members of the classes which we introduce and denote by $\left\{\kappa_{a}\right\},\{\kappa\},\{T\}$, particular members of which may be obtained by selecting $R$ from among all of the functions for which $(2 R \lambda)^{1 / 2} d u$ is an absolute invariant of $C_{\lambda}$. In this section a general member of each of the classes $\left\{\kappa_{a}\right\}$ and $\{\kappa\}$ will be characterized geometrically, and a general member of a new class of projective torsions $\{\bar{T}\}$ will be introduced and characterized geometrically. Each of these general invariants will be designated by the name of its corresponding original invariant together with the added phrase "with respect to the form $d s=(2 R \lambda)^{1 / 2} d u$." The members of the class $\{\bar{T}\}$ will be shown to be very closely related to corresponding members of the class $\{T\}$. However, a purely geometric interpretation for a general member of $\{T\}$ has not been discovered by the author.

(3) It should be observed that these differential invariants are not the usual ones unless $R=\beta \gamma$. If this restriction is removed they become extensions of the usual ones.

(4) See E. Bompiani, "I fondamenti geometrici della teoria proiettiva delle curve e delle superficie," Appendix II of Geometria Proiettiva Differenziale, G. Fubini and E. Čech [1, pp. 691-697]. 
Consider the conic $\mathcal{C}$ whose equation is (3.5) referred to the triangle whose vertices are $x, \rho, \sigma$. The lines which are tangent to $\mathcal{C}$ at the points in which the tangent to $C_{\lambda}$ at $x$ intersects $C$ will be called the $C_{\lambda}$-associates of the line $l$ joining $\rho, \sigma$ and will be denoted by $l_{\lambda,+}$ and $l_{\lambda,-}$. Their respective equations referred to the triangle whose vertices are $x, \rho, \sigma$ are easily found to be

$$
\begin{aligned}
& x_{1}+(2 R \lambda)^{1 / 2} x_{2}+(2 R / \lambda)^{1 / 2} x_{3}=0, \\
& x_{1}-(2 R \lambda)^{1 / 2} x_{2}-(2 R / \lambda)^{1 / 2} x_{3}=0 .
\end{aligned}
$$

The crossjoins of the intersections of the lines $l_{\lambda,+}$ and $l_{\lambda,-}$ with the $u$ - and $v$-tangents of $S$ at $x$ will be called the $\overline{\mathcal{C}}_{\lambda}$-associates of $l$ and will be denoted by $\bar{l}_{\lambda,+}$ and $\bar{l}_{\lambda, \ldots}$. Their respective equations are

$$
\begin{aligned}
& x_{1}-(2 R \lambda)^{1 / 2} x_{2}+(2 R / \lambda)^{1 / 2} x_{3}=0, \\
& x_{1}+(2 R \lambda)^{1 / 2} x_{2}-(2 R / \lambda)^{1 / 2} x_{3}=0 .
\end{aligned}
$$

Let $l_{2}^{\prime}$ denote the line joining the points $x$ and $x^{\prime \prime}$ and let $l_{2}$ denote its reciprocal with respect to $S$ at $x$. The $R_{\lambda}$-correspondent $\left.{ }^{5}\right)$ of the tangent to $C_{\lambda}$ at $x$ intersects the $R$-harmonic line, the $\overline{\mathcal{C}}_{\lambda}$ associates of the $R$-harmonic line, and the line $l_{2}$ in the points which we denote by $K_{1}, \bar{K}_{ \pm}, K_{2}$, respectively. Referred to the triangle whose vertices are the points $x, x_{u}, x_{v}$, the equations for the above named lines are, respectively, $\gamma \lambda^{2} x_{3}+\beta x_{2}=0, x_{1}=0,(7.6)$ and (7.7), and $x_{1}+d x_{2}+c x_{3}=0$ where $c$ and $d$ are given by (4.3). The local coordinates of $K_{1}, \bar{K}_{+}, \bar{K}_{-}, K_{2}$ are easily found to be $\left(0, \gamma \lambda^{2},-\beta\right),\left([2 R \lambda]^{1 / 2}\left[\gamma \lambda^{3}+\beta\right] / \lambda\right.$, $\left.\gamma \lambda^{2},-\beta\right),\left(-[2 R \lambda]^{1 / 2}\left[\gamma \lambda^{3}+\beta\right] / \lambda, \gamma \lambda^{2},-\beta\right),\left(-d \gamma \lambda^{2}+c \beta, \gamma \lambda^{2},-\beta\right)$, respectively. Since

$$
-d \gamma \lambda^{2}+c \beta=\left(\lambda^{\prime}+\theta_{v} \lambda^{2}-\theta_{u} \lambda\right)\left(\gamma \lambda^{3}+\beta\right) / 4 \lambda^{2},
$$

the cross ratios $\left(x, K_{1}, K_{ \pm}, K_{2}\right)$ may be found by evaluating $\left(\infty, 0, \pm[2 R \lambda]^{1 / 2}\right.$;, $\left.\left[\lambda^{\prime}+\theta_{v} \lambda^{2}-\theta_{u} \lambda\right] / 4 \lambda\right)$ to be $\pm\left(\lambda^{\prime}+\theta_{v} \lambda^{2}-\theta_{u} \lambda\right) / 4 \lambda(2 R \lambda)^{1 / 2}$. But the expression for the asymptotic curvature of $C_{\lambda}$ at $x$ with respect to the form $(2 R \lambda)^{1 / 2} d u$ becomes

$$
\left(\lambda^{\prime}+\theta_{v} \lambda^{2}-\theta_{u} \lambda\right) / 2 \lambda(2 R \lambda)^{1 / 2}, \quad \theta=\log R,
$$

when $u$ is regarded as the independent variable. Thus we have proved

THEOREM 7.1. The asymptotic curvature of $C_{\lambda}$ at $x$ with respect to the form $d s=(2 R \lambda)^{1 / 2} d u$ is characterized by the cross ratio equations $\kappa_{a}=2\left(x, K_{1}, \bar{K}_{+}, K_{2}\right)$ $=-2\left(x, K_{1}, \bar{K}_{-}, K_{2}\right)$.

The expression for the projective curvature of $C_{\lambda}$ at $x$ with respect to the form $d s=(2 R \lambda)^{1 / 2} d u$ becomes

$$
\left(\lambda^{\prime}+\beta-\theta_{u} \lambda+\theta_{v} \lambda^{2}-\gamma \lambda^{3}\right) / 2 \lambda(2 R \lambda)^{1 / 2}
$$

(5) See P. O. Bell [2, p. 392] and The $R_{\lambda}$-correspondent of the tangent to an arbitrary curve of a non-ruled surface, Bulletin of the American Mathematical Society, vol. 47 (1941), pp. 509-511. 
when $u$ is regarded as the independent variable. To characterize this invariant let $M_{1}, \bar{M}_{ \pm}, M_{2}$ denote the intersections of the tangent to $C_{-\lambda}$ at $x$ with the $R$-harmonic line, the $\bar{C}_{\lambda}$-associates of the $R$-harmonic line, and the line $l_{2}$, respectively. The local coordinates of $M_{1}, \bar{M}_{ \pm}, M_{2}$ are readily found to be $(0,1,-\lambda),\left( \pm 2[2 R \lambda]^{1 / 2}, 1,-\lambda\right),\left(\left[\lambda^{\prime}+\beta-\theta_{u} \lambda+\theta_{v} \lambda^{2}-\gamma \lambda^{3}\right] / 2 \lambda, 1,-\lambda\right)$. Hence the values of the cross ratios $\left(x, M_{1}, \bar{M}_{ \pm}, M_{2}\right)$ are equal to $\pm\left(\lambda^{\prime}+\beta-\theta_{u} \lambda\right.$ $\left.+\theta_{v} \lambda^{2}-\gamma \lambda^{3}\right) / 4 \lambda(2 R \lambda)^{1 / 2}$. Therefore

THEOREM 7.2. The projective curvature of $C_{\lambda}$ at $x$ with respect to the form $d s=(2 R \lambda)^{1 / 2} d u$ is characterized by the cross ratio equations $\kappa=2\left(x, M_{1}, \bar{M}_{+}, M_{2}\right)$ $=-2\left(x, M_{1}, \bar{M}_{-}, M_{2}\right)$.

The curvilinear differential equation for the extremal curves of the integral invariant $\int(2 R \lambda)^{1 / 2} d u$ is easily found to be

$$
\lambda^{\prime}+\theta_{v} \lambda^{2}-\theta_{u} \lambda=0, \quad \theta=\log R .
$$

We shall call these curves the projective geodesics of the surface with respect to the form $d s=(2 R \lambda)^{1 / 2} d u$. The projective geodesics of Fubini are obtained by putting $R=\gamma \beta$ in $(7.10)$.

The curvilinear differential equation for the union curves of the $R$-conjugate congruence is found by analogy with (7.10) to be

$$
\lambda^{\prime}+\beta-\theta_{u} \lambda+\theta_{v} \lambda^{2}-\gamma \lambda^{3}=0, \quad \theta=\log R .
$$

If we consider the forms (7.8) and (7.9) in connection with equations (7.10) and (7.11), respectively, we have at once

THeOREm 7.3. A curve $C_{\lambda}$ is a projective geodesic of $S$ with respect to a form $d s=(2 R \lambda)^{1 / 2} d u$ if and only if at each of its points its asymptotic curvature with respect to the same form vanishes.

TheOREM 7.4. A curve $C_{\lambda}$ is a union curve of an $R$-conjugate congruence if and only if at each of its points its projective curvature with respect to the form $d s=(2 R \lambda)^{1 / 2} d u$ vanishes.

Let $\bar{l}_{2, \pm}^{\prime}$ denote the reciprocals with respect to $S$ of the $\overline{\mathcal{C}}_{\lambda}$-associates of the line $l_{2}$, and $l_{3}^{\prime}$ denote the line joining the points $x$ and $x^{\prime \prime \prime}$. Let $\bar{\pi}_{2, \pm}$ and $\pi_{3}$ denote the planes which pass through the respective lines $\bar{l}_{2, \pm}^{\prime}$ and $l_{3}^{\prime}$ and which have the tangent to $C_{\lambda}$ at $x$ as common intersection. Let $\pi$ and $\pi_{2}$ denote, respectively, the tangent plane to $S$ at $x$ and the osculating plane of $C_{\lambda}$ at $x$. The expression defined by the cross ratio equations

$$
\bar{T}=\left(\pi_{2}, \pi, \pi_{3}, \bar{\pi}_{2,+}\right)=-\left(\pi_{2}, \pi, \pi_{3}, \bar{\pi}_{2,-}\right)
$$

is an absolute differential invariant of $C_{\lambda}$ of the third order. Because of the suitability of this invariant as a projective substitute for torsion, the author 
proposes that it be called the projective torsion of $C_{\lambda}$ at $x$ with respect to the form $d s=(2 R \lambda)^{1 / 2} d u$.

The polar reciprocals of the planes $\pi_{2}, \pi, \pi_{3}, \bar{\pi}_{2, \pm}$ with respect to a quadric of Darboux are the points $M_{2}, x, M_{3}, \bar{M}_{2, \pm}$ all of which lie on the tangent to $C_{-\lambda}$ at $x$. The points $M_{2}, M_{3}$ are the intersections of this tangent with the lines $l_{2}, l_{3}$ which are the reciprocals of $l_{2}^{\prime}, l_{3}^{\prime}$ with respect to a quadric of Darboux, and the points $\bar{M}_{2, \pm}$ are the intersections of this tangent with the $\bar{C}_{\lambda}$-associates of $l_{2}$. Since the cross ratio of four planes is equal to that of the four points which are their polar reciprocals with respect to a quadric of Darboux, the expression for $\bar{T}$ may be obtained by evaluating the cross ratio $\left(M_{2}, x, M_{3}, \bar{M}_{2,+}\right)$. Making use of the local coordinates of $M_{2}, x, M_{3}, \bar{M}_{2, \pm}$, respectively, $(M / 2 \lambda, 1,-\lambda),(\infty, 1,-\lambda),\left(2 \lambda\left[\Pi-\chi \lambda^{2}-d^{\prime}+c^{\prime} \lambda-c \beta+d \gamma \lambda^{2}\right.\right.$ $\left.\left.+d \theta_{u}-c \theta_{v} \lambda^{2}\right] /\left[\gamma \lambda^{3}+\beta\right], 1,-\lambda\right),\left(M / 2 \lambda \pm 2[2 R \lambda]^{1 / 2}, 1,-\lambda\right)$, wherein $M=\lambda^{\prime}$ $+\beta-\theta_{u} \lambda+\theta_{v} \lambda^{2}-\gamma \lambda^{3}, \Pi=p+\beta \theta_{v}+\beta_{v}, \chi=q+\gamma \theta_{u}+\gamma_{u}, \theta=\log R$, we find

$$
\begin{aligned}
\bar{T}=\left[2 \lambda \left(M^{\prime}\right.\right. & \left.+2 \Pi \lambda-2 \chi \lambda^{3}\right) \\
& \left.-M\left(3 \lambda^{\prime}+\beta+\theta_{u} \lambda+\theta_{v} \lambda^{2}+\gamma \lambda^{3}\right)\right] / 4 \lambda(2 R \lambda)^{1 / 2}\left(\gamma \lambda^{3}+\beta\right) .
\end{aligned}
$$

The invariant $T$ given by (7.3) with $\beta \gamma$ replaced by a function $R$ may be shown to be related to the author's invariant $\bar{T}$ by the simple formula

$$
\bar{T} d \sigma=T d s,
$$

in which $d \sigma$ is the projective linear element defined by $d \sigma=\left(\beta d u^{3}+\gamma d v^{3}\right) / d u d v$ and $d s$ is the generalized element of projective arc length defined by $d s=(2 R \lambda)^{1 / 2} d u$.

If at a general point $x$ of a curve $C_{\lambda}$ the osculating plane of $C_{\lambda}$ contains the point $x^{\prime \prime \prime}$, the osculating plane is stationary and the curve $C_{\lambda}$ is a plane curve. In this case the planes $\pi_{2}$ and $\pi_{3}$ coincide at every point of $C_{\lambda}$; and $\bar{T}$, therefore, vanishes identically along $C_{\lambda}$. Moreover, equation (7.13) shows that if $C_{\lambda}$ is not an asymptotic curve, $\bar{T}$ can vanish identically along $C_{\lambda}$ only if its numerator does. If the numerator of $\bar{T}$ vanishes identically along $C_{\lambda}$, the points $M_{2}, M_{3}$ which correspond to a general point of $C_{\lambda}$ must coincide. Hence, their polar reciprocals $\pi_{2}, \pi_{3}$ must also coincide and the osculating plane $\pi_{2}$ is, therefore, stationary. To summarize:

TheOREM 7.5. A curve $C_{\lambda}$ is a plane curve if and only if at a general one of its points its projective torsion with respect to the form $d s=(2 R \lambda)^{1 / 2} d u$ is equal to zero.

The equation obtained by setting the numerator of the expression for $\bar{T}$ equal to zero is the curvilinear differential equation for all of the plane curves of $S$.

It may be observed that although all of the results up to this point have been developed in association with a general form $d s=(2 R \lambda)^{1 / 2} d u$, a considerable number of them are, clearly, independent of $R$. 
8. Special conjugate congruences. In a recent paper $[1$, p. 681$]$ the author has shown that the $R$-conjugate line may be characterized geometrically in association with the curves which a pair of covariant points $\omega_{1}, \omega_{2}$ describe as $x$ moves along $C_{\lambda}$. To complete this characterization for special cases of the $R$-conjugate line, corresponding pairs of points $\omega_{1}, \omega_{2}$ must be located.

The projective normal is the special case of the $R$-conjugate line which may be defined in association with the points $\omega_{1}, \omega_{2}$ given by

$$
\omega_{1}=\tau-2 a^{\prime} b y, \quad \omega_{2}=\tau+2 a^{\prime} b y
$$

wherein $\tau=y_{u v}-\alpha y_{u}-\beta y_{v}+\alpha \beta y$. Geometric determinations, which do not depend on the definitions of the quadrics of Wilczynski and Lie, have been given by the author $[2$, p. 392] for these points. These determinations supplement the method of $[1$, p. 681$]$ to complete a simple geometric determination of the projective normal.

The following considerations serve to introduce another important special case of the $R$-conjugate line. Let $l_{k}$ denote a general line of the first canonical pencil. The line $l_{k}$ intersects the tangents of $S$ at $y$ to the asymptotic $u$-and $v$-curves in the points $\rho, \sigma$ defined in (2.2) where

$$
\begin{aligned}
& \beta=\left(\log a^{\prime 2} b\right)_{u} / k-\left(\log a^{\prime} b\right)_{u} / 2, \\
& \alpha=\left(\log b^{2} a^{\prime}\right)_{v} / k-\left(\log a^{\prime} b\right)_{v} / 2 .
\end{aligned}
$$

The lines $l_{2}, l_{3}, l_{4}$ and $l_{\infty}$ are the first directrix of Wilczynski, the reciprocal of the axis of $\dot{C} e c h$, the first canonical edge of Green, and the reciprocal of the projective normal, respectively. As $y$ moves over $S$ the points $\rho, \sigma$ of $l_{k}$ generate transversal surfaces $S_{\rho}$ and $S_{\sigma}$ of the congruence described by $l_{k}$. The $v$-tangent at $\rho$ to $S_{\rho}$ intersects $l_{k}^{\prime}$, the reciprocal of $l_{k}$, in the point which we denote by $\eta_{k}$ whose coordinates are given by $\eta_{k}=\tau-\beta_{v} y$, where $\alpha, \beta$ are the functions (8.2). Likewise the $u$-tangent at $\sigma$ to $S_{\sigma}$ intersects $l_{k}^{\prime}$ in the point which we denote by $\xi_{k}$ whose coordinates are given by $\xi_{k}=\tau-\alpha_{u} y$. If we let $\omega_{1}=\eta_{k}, \omega_{2}=\xi_{k}$, we have $\omega_{1}-\omega_{2}=\left(\log b / a^{\prime}\right)_{u v} y / k$. Hence we have

THEOREM 8.1. If the fundamental points $\omega_{1}, \omega_{2}$ are chosen as the points $\eta_{k}$, $\xi_{k}$ on a canonical line $l_{k}^{\prime}$ of the second kind, the associated $R$-conjugate line is independent of $k$ and joins the points $y, \tau$ defined by (2.2) wherein the associated functions $\alpha, \beta$ are given by $\alpha=-(\log R)_{v} / 2, \beta=-(\log R)_{u} / 2$ with $R=\left(\log b / a^{\prime}\right)_{u v}$.

Consider the following problem. Find an integral of the form $\int(2 R \lambda)^{1 / 2} d u$ with the following properties: (i) it is absolutely invariant under the most general transformation of variables which maintains the parametric character of the asymptotic net of $S$ and the metric properties of any orthogonal cartesian coordinate system to which $S$ may be referred, (ii) the normal is the cusp axis of $x$ with respect to the extremal curves of the integral. 
This problem is found to possess a unique solution (except for an unessential arbitrary constant factor). Let any orthogonal system of cartesian coordinates be established in ordinary metric space. Let $x$ denote a vector from the origin to a point on the surface $S$ wherein the coordinates $x^{(i)},(i=1,2,3)$, are measured with respect to equal units on the three axes. Let the asymptotic net be parametric. Let $X$ denote the unit normal to $S$ at $x$. The first fundamental form is given by $E d u^{2}+2 F d u d v+G d v^{2}$ where $E, F, G$ are defined by the scalar products

$$
E=\left(x_{u} \mid x_{u}\right), \quad F=\left(x_{u} \mid x_{v}\right), \quad G=\left(x_{v} \mid x_{v}\right) .
$$

The second fundamental form is given by $2 D^{\prime} d u d v$ where $D^{\prime}=\left(X \mid x_{u v}\right)$. The fundamental differential equations which are satisfied by $x^{(i)}, X^{(i)}$ are known to be

$$
\begin{aligned}
& x_{u u}=\{11,1\} x_{u}+\{11,2\} x_{v}, \\
& x_{u v}=\{21,1\} x_{u}+\{12,2\} x_{v}+D^{\prime} X, \\
& x_{v v}=\{22,1\} x_{u}+\{22,2\} x_{v}, \\
& X_{u}=F D^{\prime} x_{u} / H^{2}-E D^{\prime} x_{v} / H^{2}, \\
& X_{v}=-G D^{\prime} x_{u} / H^{2}+F D^{\prime} x_{v} / H^{2}, \quad H^{2}=E G-F^{2},
\end{aligned}
$$

where $\{i k, l\}$ denote the Christoffel symbols $\left({ }^{6}\right)$ of the second kind for the first fundamental form.

The equation of Gauss,

$$
H K=(H\{11,2\} / E)_{v}-(H\{12,2\} / E)_{u},
$$

where $K$ is the total curvature defined by $K=-D^{\prime 2} / H^{2}$, and the equations of Codazzi,

$$
D_{u}^{\prime}-(\{11,1\}-\{12,2\}) D^{\prime}=0, \quad D_{v}^{\prime}-(\{22,2\}-\{21,1\}) D^{\prime}=0,
$$

are the three integrability conditions of (8.3). that

By means of the definitions of the Christoffel symbols it is easy to show

$$
(\log H)_{u}=\{11,1\}+\{12,2\}, \quad\left(\log D^{\prime}\right)_{v}=\{22,2\}-\{21,1\} .
$$

Making use of these identities and the Codazzi equations (8.5) to solve for $\{11,1\},\{12,2\},\{22,2\}$ and $\{21,1\}$ in terms of $H$ and $D^{\prime}$, we obtain the following form for the first three differential equations of (8.3):

$$
\begin{gathered}
x_{u u}=\theta_{u} x_{u}+\{11,2\} x_{v}, \quad x_{u v}=\nu_{v} x_{u}+\nu_{u} x_{v}+D^{\prime} X, \\
x_{v v}=\{22,1\} x_{u}+\theta_{v} x_{v},
\end{gathered}
$$

where $\theta=\log \left(H D^{\prime}\right)^{1 / 2}, \nu=\log \left(H / D^{\prime}\right)^{1 / 2}$.

(6) See, e.g., E. P. Lane [1, p. 222]. 
The homogeneous cartesian coordinates $x^{(1)}, x^{(2)}, x^{(3)}, 1$ of $x$ may be regarded as special projective coordinates of $x$ which are referred to the tetrahedron of reference whose faces are the three cartesian coordinate planes and the plane at infinity. The unit point must, of course, be properly selected so that the system is cartesian. Let us determine the transformation $\mu x=\bar{x}$ which converts homogeneous cartesian coordinates $x$ into coordinates $\bar{x}$ which satisfy the differential equations in the unique $R$-canonical form whose associated $R$-conjugate line is the normal to $S$ at $\bar{x}$. Differentiating the equation $\bar{x}=\mu x$ we find $\bar{x}_{u}=\mu x_{u}+\mu_{u} x, \bar{x}_{v}=\mu x_{v}+\mu_{v} x, \bar{x}_{u v}=\mu x_{u v}+\mu_{v} x_{u}+\mu_{u} x_{v}+\mu_{u v} x$. Let us choose $\mu$ in such a way that $\bar{x}_{u v}$ lies on the normal to $S$ at $x$. The point $x_{u v}-\nu_{v} x_{u}-\nu_{u} x_{v}$ is the point at infinity on the normal to $S$ at $x$, for its fourth coordinate is, clearly, zero and the second equation of (8.6) shows that its first three coordinates are direction numbers of the normal to $S$ at $x$. The coordinates of any selected point on the normal to $S$ at $x$ may be represented by a suitably chosen linear combination of the corresponding coordinates of the points $x$ and $x_{u v}-\nu_{v} x_{u}-\nu_{u} x_{v}$. The point $\bar{x}_{u v}$ will therefore lie on the normal to $S$ at $x$ if and only if $\mu$ satisfies the equations $(\log \mu)_{v}=-\nu_{v},(\log \mu)_{u}=-\nu_{u}$. Integrating these we obtain $\mu=k\left(D^{\prime} / H\right)^{1 / 2}, k=$ arbitrary constant. The effect of the transformation $\bar{x}=k\left(D^{\prime} / H\right)^{1 / 2} x$ on the first and third equations of (8.6) is to produce a system of the form (2.4) wherein

$$
\begin{aligned}
\theta= & \log \left(D^{\prime 3} / H\right)^{1 / 2}, \quad \beta=\{11,2\}, \quad \gamma=\{22,1\}, \\
p= & {\left[\left(H_{u} / H\right)^{2}-\left(D_{u}^{\prime} / D^{\prime}\right)^{2}\right] / 4+\{11,2\}\left[\log H / D^{\prime}\right]_{v} / 2 } \\
& -\left[\left(H / D^{\prime}\right)^{1 / 2}\right]_{u u} /\left(H / D^{\prime}\right)^{1 / 2}, \\
q= & {\left[\left(H_{v} / H\right)^{2}-\left(D_{v}^{\prime} / D^{\prime}\right)^{2}\right] / 4+\{22,1\}\left[\log H / D^{\prime}\right]_{u} / 2 } \\
& -\left[\left(H / D^{\prime}\right)^{1 / 2}\right]_{v v} /\left(H / D^{\prime}\right)^{1 / 2} .
\end{aligned}
$$

The normal is therefore the $R$-conjugate line for which $R=k\left(D^{\prime 3} / H\right)^{1 / 2}, k=$ arbitrary const. Since, moreover, $K=-D^{\prime 2} / H^{2}$, we have $R=c K^{1 / 4} D^{\prime}, c=$ arbitrary const. The differential form $2 K^{1 / 4} D^{\prime} d u d v$ is absolutely invariant under all transformations which maintain the orthogonal cartesian properties of the coordinate system and the parametric character of the asymptotic net. Both the total curvature $K$ of $S$ at $x$ and the second fundamental form $2 D^{\prime} d u d v$ are intrinsically connected with $S$ at $x$. Let us recall that if we consider a point $x(s+\Delta s)$ near to $x(s)$, where $s$ denotes length of arc along a curve $C_{\lambda}$, for an arbitrarily selected parametric net, the associated second fundamental form of $S$ is equal in absolute value to twice the principal part of the distance from the point $x(s+\Delta s)$ to the plane which is tangent to $S$ at $x(s)$.

The above results in combination with Theorem (2.3) [1, p. 681] yield the following

THEOREM 8.2. If the surface $S$ is referred to its asymptotic net as parametric, 
the normal to $S$ at a non-parabolic point is the cusp axis of $x$ with respect to the extremal curves of the integral invariant

$$
\int\left(2 K^{1 / 4} D^{\prime} d u d v\right)^{1 / 2}
$$

where $2 D^{\prime} d u d v$ is the second fundamental form of $S$ and $K$ is the total curvature of $S$ at $x$.

\section{REFERENCES}

V. G. Grove

1. On canonical forms of differential equations, Bulletin of the American Mathematical Society, vol. 36 (1930), pp. 582-586.

P. O. BELL

1. Projective analogues of the congruence of normals, American Journal of Mathematics, vol. 62 (1940), pp. 680-686.

2. A study of curved surfaces by means of certain associated ruled surfaces, these Transactions, vol. 46 (1939), pp. 389-409.

N. Abramescu

1. Nouvelle méthode pour obtenir la cubique qui donne les tangentes de Darboux en un point d'une surface, Comptes Rendus de l'Académie des Sciences, Paris, vol. 209 (1939), pp. 780-781.

E. J. WiLCZYNSKI

1. Projective differential geometry of curved surfaces (first memoir), these Transactions, vol. 8 (1907), pp. 233-260.

2. Projective differential geometry of curved surfaces (second memoir), these Transactions, vol. 9 (1908), pp. 79-120.

G. M. GREeN

1. Memoir on the general theory of surfaces and rectilinear congruences, these Transactions, vol. 20 (1919), pp. 79-153.

2. Nets of space curves, these Transactions, vol. 21 (1921), pp. 207-236.

FUBiNi AND ČECH

1. Geometria Proiettiva Differenziale, vol. 1, 1926; vol. 2, 1927; Bologna, Zanichelli.

E. P. LANE

1. Projective Differential Geometry of Curves and Surfaces, The University of Chicago Press, 1932.

UNIVERSITY OF KANSAS,

LAWRENCE, KANS. 